\title{
A Model for Dark Energy Decay
}

\author{
Elcio Abdalla* and L. L. Grae円 \\ Instituto de Física, Universidade de São Paulo, CP 66318, 05315-970, São Paulo, Brazil \\ Bin Wang ${ }^{7}$ \\ INPAC and Department of Physics, Shanghai Jiao Tong University, 200240 Shanghai, China
}

\begin{abstract}
We discuss a model of non perturbative decay of dark energy. We suggest the possibility that this model can provide a mechanism from the field theory to realize the energy transfer from dark energy into dark matter, which is the requirement to alleviate the coincidence problem. The advantage of the model is the fact that it accommodates a mean life compatible with the age of the universe. We also argue that supersymmetry is a natural set up, though not essential.
\end{abstract}

PACS numbers:

\section{INTRODUCTION}

It is a rather accepted fact that our universe contains about $70 \%$ dark energy (DE), $25 \%$ cold dark matter $(\mathrm{DM})$ and a remaining fraction of baryonic matter [1. In the concordance model the cosmological constant is the easiest explanation for the DE. However, it is difficult to understand the cosmological constant in terms of fundamental physics. Its observed value is too small, a fact referred to as the cosmological constant problem. The fact that the amount of DE and of DM are of the same order of magnitude today is neither easy to comprehend, as we know that in the past they differed by several orders of magnitude. This is known as the coincidence problem.

An interaction in the dark sector leads to a mechanism to alleviate the coincidence problem 22. Moreover, in the framework of field theory, it is inevitable to consider an interaction between $\mathrm{DM}$ and $\mathrm{DE}$, given that they are fundamental fields of the theory [3]. The dark sector interaction has been widely discussed in the literature [4-12]. Extensive analysis using the WMAP, SNIa, BAO and SDSS data etc has been performed in refs. [5, as well as the use of the late ISW effect to probe the coupling between dark sectors 6 .

A change in the growth index was found in refs. 8, 9, as a consequence of the interaction, as well as further consequences concerning the growth of cosmic structure 8]-12. More recently, the interaction has been seen as an external potential leading to observable corrections to the Layser Irvine equation [11, 12. As a consequence, a small positive coupling has been tightly constrained 12 in agreement with the results given in [6] from CMB. The small positive coupling indicates that there is energy transfer from DE to DM, which helps alleviate the coincidence problem 6, 7].

Another possible explaination for the universe acceler-

\footnotetext{
*Electronic address: eabdalla@fma.if.usp.br

${ }^{\dagger}$ Electronic address: leilagraef@usp.br

‡Electronic address: wang b@sjtu.edu.cn
}

ation is achieved in finding alternatives to the Einstein gravity. An example is the $f(R)$ gravity, constructed based on a Lagrangian density given by an arbitrary function $f(R)$ depending on the curvature scalar [13]. $f(R)$ gravity is considered as the simplest modification to Einstein's general relativity. The $f(R)$ gravity turns out to be conformally equivalent to an interaction model between DE and DM [14. In the Einstein frame, the model does not possess a standard matter-dominated epoch as in the Jordan frame, but contains the coupling between the canonical scalar field to the non-relativistic matter. It was found that the condition that $f(R)$ gravity avoids a short-timescale instability and maintains the agreement with $\mathrm{CMB}$ is exactly equivalent to the requirement of an energy flow from DE to DM in the interaction model, which ensures the alleviation of the coincidence problem in the Einstein frame [15].

\section{THE INTERACTING MODEL}

When there is an energy exchange between dark energy and dark matter, none of them is separately conserved. In such a case the conservation equations are written as

$$
\begin{gathered}
\rho_{D E}^{\prime}+3 H \rho_{D E}\left(1+w_{D E}\right)=Q_{D E}, \\
\rho_{D M}^{\prime}+3 H \rho_{D M}\left(1+w_{D M}\right)=Q_{D M},
\end{gathered}
$$

where prime denotes the derivative with respect to conformal time, $w=p / \rho$ and $Q$ is the interaction factor. We can see, through eq.(1), that we can define an effective $w$ for the dark energy as $w_{e f f}=w-\frac{Q_{D E}}{3 H \rho_{D E}}$, which accounts for the interaction.

Supposing a decay from dark energy into dark matter, it is natural to expect that phenomenologically $Q$ is proportional to the energy density of dark energy and to the decay rate $\Gamma$,

$$
Q_{D M}=-Q_{D E}=\Gamma \rho_{D E},
$$

where $\Gamma>0$ indicates an energy flow from DE to DM. By defining $\Gamma$ we can find $Q_{D E}$. We can also write the 
coupling term in the form of $Q_{D E}=-\gamma H \rho_{D E}$, where $\gamma=\Gamma / H$. Integrating equation (1) we get the evolution for the dark energy density

$$
\rho_{D E}=\rho_{D E 0} a^{-3\left(1+w_{e f f}\right)},
$$

where $w_{\text {eff }}=w+\gamma / 3$.

We know that the lifetime of the dark energy must be of the order of the age of the universe. If it was much more the effect of the interaction would be negligible, and on the other hand, if it was much less, the value of the dark energy density should have been much higher in the past, and the coupling term $Q_{D E}$, in this case, might not have the small value predicted by the observations. Moreover, as it's well known, the standard $\Lambda C D M$ model fits very well the various observational results available, so it would be nice to have a model whose dynamics could approach the dynamics of the standard model, in this case we expect a dark energy density not much higher than $10^{-47} \mathrm{GeV}^{4}$ even in the past.

We can see through eq.11 that, with a coupling, each component does not conserve separately anymore, they evolve correlated. That's why it becomes possible to reproduce a scaling solution of the kind [16]

$$
\rho_{D E}=\rho_{D M} a^{\xi}
$$

where $\xi=-3 w_{\text {eff }}$.

For the cosmological constant case, being $\rho_{D E}$ a constant, we have $\xi=3$, which suffers the coincidence problem. When $\xi=0$, the ratio $\rho_{D M} / \rho_{D E}=$ const and there is no coincidence problem [17. If there is energy decay from dark energy to dark matter, we can have $\xi<3$, which can accommodate longer period for the energy densities of dark energy and dark matter to be comparable so that to alleviate the coincidence problem.

\section{A MODEL FOR THE DECAY}

Here we propose a further model for the interaction. Suppose that a positive cosmological constant (e.g. de Sitter like cosmology) is modeled by a non zero scalar vacuum energy, and that such a non zero vacuum energy density is very small, $V_{0} \sim 10^{-47} \mathrm{GeV}^{4}$. We suppose a scalar with doubly degenerated energy minima and a small breaking term to provide such a small energy difference. This is indeed very rare and generally unnatural except for a well known case, that is if there is a symmetry forcing the vacua to be equal and a nonperturbative break of that symmetry. There actually exists such a theoretical model. The Wess Zumino model [18 has a set of degenerated bosonic vacua as a consequence of supersymmetry which presumably is broken only non perturbatively. We thus suppose this is the case and consider a bosonic potential

$$
V(\phi)=\left|2 m \phi-3 \lambda \phi^{2}\right|^{2}+Q(\phi) \equiv U(\phi)+Q(\phi),
$$

where $\phi=\varphi+i B$ is a complex scalar of mass $m$ and coupling $\lambda$. The first term, $U(\phi)$, corresponds to the bosonic sector of the Wess-Zumino Lagrangian and $Q(\phi)$ is a supersymmetry breaking term of power law type. The term $Q(\phi)$ is adjusted so that we have the cosmological constant value at the metastable minimum. The exact form of the breaking term, however, is not needed for the computations.

This potential has a set of zeros, in $\varphi=0$, and at $\varphi=\frac{2 m}{3 \lambda}$, if $B=0$. Let us suppose that we have only one (uncharged) bosonic field, so $B=0$. There is an interaction with a fermion which for our calculations here is irrelevant. It should become here clear that supersymmetry is not a requirement for the present work, its just a motivation for a potential with such a form. The potential for this model is illustrated in Fig.1.

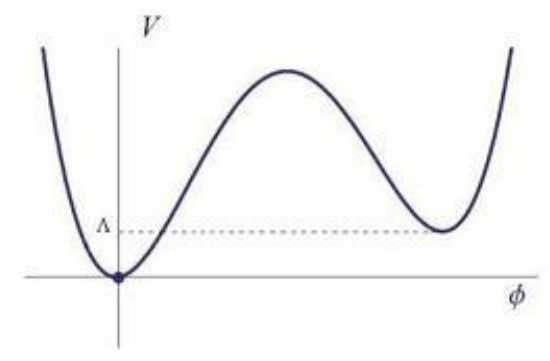

Figure 1: The Potential of the Field $\phi$.

The physical mechanism is supposed to run as follows. The field at the false vacuum represents the dark energy. We know, however, that there can be a decay from the false to the true vacuum. After the field passes the potential barrier its equation of state is no longer that of a dark energy, and there is a non negligible kinetic energy. As happens in the old inflationary scenario, the transition to the true vacuum occurs through the formation of bubbles of new vacuum. After these bubbles are nucleated, they begin to expand very fast, as the energy of the transition accelerates the bubble wall 19. At some moment, however, the walls of these bubbles, which carries the energy of the transition, begin to collide. Through this process, in the end, the energy released in the conversion of the false vacuum into the true vacuum can produce a kind of new component.

Since our field has negligible couplings to the baryons (which is a reasonable supposition since we have never detected such interaction), we expect that the final product of the transition must correspond to the dark sector. However, we are considering a supersymmetric potential, so we still have an interaction term $g \phi \bar{\psi} \psi$ (where $g$ is a coupling constant), which describes the talk between the scalar field and the fermionic field. So this new component produced can be pressureless fermionic cold dark matter. However since the decay time from the metastable vacuum to the stable vacuum of the scalar potential is of the order of the lifetime of the universe, the dark matter produced in $\phi$ decay is not the dominant 
dark matter in the universe. In order for the standard cosmology to be recaptured, there has to be another dominant component of dark matter that was around high redshift, $z \sim 1000$.

In the following we will compute the decay from the metastable vacuum to the stable vacuum of the scalar potential during the lifetime of the universe. For certain values of the parameters, the barrier has the exact height needed for a decay occurring during this time.

\section{COMPUTATION OF THE DECAY RATE}

The decay rate (per unit volume) from the metastable to the stable minimum of the potential $V(\varphi)$ is given, according to the semiclassical method, by [19]

$$
\frac{\Gamma}{V}=\frac{S_{E}^{2}(\tilde{\varphi}(\rho))}{(2 \pi \hbar)^{2}} \times e^{-\left(\frac{S_{E}}{\hbar}-\frac{S_{\Lambda}}{\hbar}\right)} \times\left(\frac{\operatorname{det}^{\prime}\left(-\partial_{\mu} \partial_{\mu}+V^{\prime \prime}(\tilde{\varphi}(\rho))\right.}{\operatorname{det}\left(-\partial_{\mu} \partial_{\mu}+V^{\prime \prime}\left(\varphi_{+}\right)\right)}\right)^{-\frac{1}{2}}
$$

where $\varphi_{+}$is the value of the field at the false vaccum. $\tilde{\varphi}(\rho)$ corresponds, in analogy with the case of particles, to the classical path in Euclidean space crossing the potential $-V(\varphi)$ with the boundary conditions $\varphi_{\text {initial }}=\varphi_{\text {final }}=\varphi_{+}$. The euclidean action $S_{E}$ in the above expression corresponds, in this analogy, to the action of a particle in this oscilating trajectory, and it is evaluated in relation to the action of a particle at the false vacuum, $S_{\Lambda}$. The determinant is defined as a ratio with respect to the determinant at the false vacuum, which has the effect of a normalization.

The calculation of the decay rate is complicated but standard, a task usually without any analytic solution. We use here the so called thin wall approximation [19], in which the energy difference between the two minima, given by the parameter $\epsilon$, is small, and we can make the calculations perturbatively in $\epsilon$, thus leading to an analytical solution.

The classical equation of motion, in the euclidean space, of the field $\varphi$ described by the potential $V(\varphi)$, is obtained by minimizing the action

$$
\frac{\delta S_{E}(\varphi(x))}{\delta \varphi}=\left(-\partial_{\mu} \partial_{\mu} \varphi(x)+V^{\prime}(\varphi)\right)=0 \quad .
$$

This is exactly the equation of motion of a scalar field in a potential $-V(\varphi)$ in Minkowski space.

We suppose the boundary condition

$$
\lim _{\tau \rightarrow \pm \infty} \varphi(\vec{x}, \tau)=\varphi_{+} .
$$

Due to the symmetry of the problem we can assume the solution to be euclidian invariant, thus, $\varphi(\vec{x}, \tau) \rightarrow$ $\varphi\left(\left(\mid \overrightarrow{\left.x\right|^{2}}+\tau^{2}\right)^{\frac{1}{2}}\right)$. For convenience we can define the variable $\rho=\left(|\vec{x}|^{2}+\tau^{2}\right)^{\frac{1}{2}}$. In this case the equation of motion becomes

$$
\frac{\partial^{2} \varphi}{\partial \rho^{2}}+\frac{3}{\rho} \frac{\partial}{\partial \rho} \varphi-V^{\prime}(\varphi)=0
$$

This equation of motion for the field $\varphi$ is analog to the equation of motion of a particle at position $\varphi$, moving in a time $\rho$, subject to a potential $-V(\varphi)$. The second term has a form analogous to a friction term. Observing the symmetry of the problem it is easy to see that the decay occurs by the formation of bubbles of true vacuum surrounded by the false vacuum outside. The term $\frac{\partial \varphi}{\partial \rho}$ is different from zero only at the bubble wall, since the field is at rest inside and outside. If this wall is thin we can consider $\rho=R$ in this region ( $R$ denotes de radius of the bubble). When $R$ is very large, as occurs when the energy difference $\epsilon$ is small, we can neglect the friction term, as it is multiplied by $1 / \rho$ that is equal to $1 / R$ in the wall. So the equation of motion becomes

$$
\frac{\partial^{2} \varphi}{\partial \rho^{2}}=V^{\prime}(\varphi)
$$

The calculation of the action can be separated in three regions: outside the bubble, at the thin wall and inside the bubble. In each of these regions the corresponding value of the field is

$$
\begin{aligned}
& \varphi=2 m / 3 \lambda, \text { if } 0<\rho \ll R, \\
& \varphi=\tilde{\varphi}, \quad \text { if } \quad \rho \approx R, \\
& \varphi=0, \quad \text { if } \rho \gg R .
\end{aligned}
$$

The exact form of $\tilde{\varphi}$ is not needed here for our purposes. So, the expression for the action can be calculated as

$$
\begin{aligned}
& S_{E}-S_{\Lambda} \approx 2 \pi^{2} \int_{0}^{R-\Delta} d \rho \rho^{3}(-\epsilon)+ \\
& 2 \pi^{2} \int_{R-\Delta}^{R+\Delta} d \rho \rho^{3}\left(\frac{1}{2}\left(\frac{d \tilde{\varphi}}{d \rho}\right)^{2}+U\right)+2 \pi^{2} \int_{R+\Delta}^{\infty} d \rho \rho^{3}(0)
\end{aligned}
$$

where $\Delta$ represents the width of the wall. After integrating we obtain the action

$$
\begin{aligned}
S_{E}-S_{\Lambda} & =-2 \pi^{2} \epsilon \frac{R^{4}}{4}+2 \pi^{2} R^{3} \int_{R-\Delta}^{R+\Delta} d \rho\left(\frac{1}{2}\left(\frac{d \tilde{\varphi}}{d \rho}\right)^{2}+U\right)+0 \\
& =-\frac{1}{2} \pi^{2} R^{4} \epsilon+2 \pi^{2} R^{3} S_{1}
\end{aligned}
$$

where we defined $\left(S_{1}=\int_{R-\Delta}^{R+\Delta} d \rho\left(\frac{1}{2}\left(\frac{d \tilde{\varphi}}{d \rho}\right)^{2}+U\right)\right)$. From now on we call $S_{E}-S_{\Lambda}$ simply as $S$. We can find $R$ by minimizing the action

$$
\frac{d S}{d R}=-2 \pi^{2} R^{3} \epsilon+6 \pi^{2} R^{2} S_{1}=0
$$

and so we obtain $R=3 S_{1} / \epsilon$. We can see that $R \rightarrow \infty$ if $\epsilon \rightarrow 0$. This is the reason why in our approximation we neglected the friction term when $\epsilon$ is very small.

Integrating equation (10), considering $\epsilon$ small, we obtain the relation $\frac{\partial}{\partial \rho} \varphi=\sqrt{2 U}$, allowing to get for $S_{1}$ the 
expression

$$
S_{1}=\sqrt{2} \int_{\varphi_{-}}^{\varphi_{+}} d \varphi \sqrt{U}
$$

Substituting the expression of the Wess-Zumino supersymmetric potential into the expression of $S_{1}$ we get

$$
S_{1}=\sqrt{2}\left\{\frac{4 m^{3}}{27 \lambda^{2}}\right\}
$$

Using above expression in eq. 12 we can see that the action will have the form

$$
S \approx \frac{m^{12}}{\lambda^{8} \epsilon^{3}}
$$

Since the exponential term in the decay rate dominates whenever we are within the validity of the semiclassical limit, the pre-exponential term will change the result so insignificantly on the scale we are working, that a simple estimate of it's order of magnitude is enough. We know that the dimension of the pre-exponential term is of $m^{4}$ and it's value is determined by the parameters of the theory with dimension of mass. Therefore we can estimate that for the energy scale we are dealing with, the decay rate per unit volume can be written as 21.

$$
\frac{\Gamma}{V}=m^{4} e^{-m^{12} /\left(\lambda^{8} \epsilon^{3}\right)} .
$$

We will simply estimate the pre-exponential terms as 1 $\mathrm{GeV}^{4}$ in order to facilitate the calculations and give the correct units we are dealing with. Its easy to show that it will not affect our results.

So, by substituting the value of $\epsilon$ and $\lambda$, we obtain for the decay rate (per volume)

$$
\frac{\Gamma}{V}=e^{-10^{156}\left(\frac{m}{G e V}\right)^{12}} G e V^{4} .
$$

Inverting the expression of the decay rate we then obtain the decay time (times the volume) of a particle. Due to the symmetry of our problem we can simply take the fourth root of the result in order to obtain the following decay time

$$
t_{\text {decay }}=10^{-25}\left\{\exp \left(10^{156}\left(\frac{m}{G e V}\right)^{12}\right)\right\}^{\frac{1}{4}} s .
$$

If we equate this decay time to the age of the universe, $\left(10^{17} s\right)$ we obtain for the mass the value

$$
m \sim 10^{-13} \mathrm{GeV} .
$$

Having this result its easy to calculate that the value of $\mathrm{R}$ (the radius of the bubbles in the moment they are formed) is about $10^{-3} \mathrm{~cm}$. After the tunneling the field evolves according to the classical field equation, which is simply the analitic continuation of the euclidean field equation (9) back to real time.

Similar results are obtained when using other potential with similar characteristics, as for example $U=$ $\frac{\lambda}{8}\left(\phi^{2}-\frac{m^{2}}{\lambda}\right)^{2}$ plus a term that generates a metastable minimum with the energy density of the cosmological constant. Using other potential of this kind we obtain as result approximately the same order of magnitude for the mass.

If we consider in our calculations the gravitational effect we must consider the following action

$$
\bar{S}=\int d^{4} x \sqrt{-g}\left(\frac{1}{2} g^{\mu \nu} \partial_{\mu} \varphi \partial_{\nu} \varphi-V(\varphi)-\frac{R}{16 \pi G}\right) \quad .
$$

It is possible to show that using the thin wall approximation we get the following relation between the action including gravity and the one we have calculated before 20],

$$
\bar{S}=\frac{S_{0}}{\left(1+\left(\frac{R_{0}}{2 \Delta}\right)^{2}\right)^{2}}
$$

where $S_{0}$ is the expression for the action obtained in the previous case, $R_{0}$ is the radius of the bubble formed in that case, $\bar{S}$ the new action considering gravity and $\Delta$ is the Schwarzschild radius associated to the bubble of new vacuum.

In the case that gravity is included the thin wall approximation is a good approximation for all cases of our interest. It's possible to show that in this case we can neglect the friction term and also the expansion of the universe.

The energy released in the conversion of false into true vacuum is proportional to the volume of the bubble of new vacuum formed. There is therefore a Schwarzschild radius associated to this energy. We can see by the equation above that if the radius of the bubble of true vacuum is comparable to its Schwarzschild radius then it is important to include gravity in our calculation [20], otherwise we recover our previous result. Equating the radius $R$ of our bubble to the expression of the Schwarzschild radius it's easy to show that, for the scale of the energies we are working with, we really could have simply neglected the gravitational effects.

Let us briefly discuss the symmetry breaking that generates the false vacuum energy density. As $\varphi \sim \frac{m}{\lambda}$ at this point, we can see that terms such as

$$
Q(\varphi)=m^{2} \varphi^{2}, \lambda m \varphi^{3}, \lambda^{2} \varphi^{4}
$$

breaks the symmetry and causes this vacuum to have the energy density corresponding to the cosmological constant.

\section{CONCLUSION}

We calculated the decay of a particle of dark energy, with mass of the order $m \sim 10^{-13} \mathrm{GeV}$, described by the potential $V(\varphi)=\left|2 m \varphi-3 \lambda \varphi^{2}\right|^{2}+Q(\varphi)$, from the metastable to the stable minimum of the potential. The timescale of such a process is compatible with the order of magnitude of the age of the universe. We suggest that such a kind of quintessence model can provide a 
mechanism, from field theory, to explain the decay of dark energy into dark matter, alleviating the coincidence problem of the concordance model of cosmology.

We think that, in a future work, a further analysis of the evolution of the bubbles of new vacuum after its formation, could give us previsions for the size and configuration of these bubbles, which could be a potentially testable signal of this model.

In view of the arguments in [15, our model can be equivalent to an $f(R)$ gravity, with a calculable function
$f(R)$. We also think it is interesting to investigate the $f(R)$ gravity based on the proposed field theory model.

\section{Acknowledgments}

The authors wish to thank CNPq and FAPESP, Brazil and NNSF, China, for support.
[1] S. J. Perlmutter et al., Nature 391 (1998) 51; A. G. Riess et al., Astron. J. 116 (1998) 1009 ; S. J. Perlmutter et al., Astroph. J. 517 (1999) 565 ; J. L. Tonry et al., Astroph. J. 594 (2003) 1; A. G. Riess et al., Astroph. J. 607 (2005)665 ; P. Astier et al., Astron. Astroph. 447 (2005) 31 ; A G. Riess et al., Astroph. J. 659 (2007) 98.

[2] L. Amendola, Phys. Rev. D 62, 043511 (2000); L. Amendola and C. Quercellini, Phys. Rev. D 68 (2003) 023514 ; L. Amendola, S. Tsujikawa and M. Sami, Phys. Lett. B 632 (2006) 155; D. Pavon, W. Zimdahl, Phys. Lett. B 628 (2005) 206, S. Campo, R. Herrera, D. Pavon, Phys. Rev. D 78 (2008) 021302(R); C. G. Boehmer, G. Caldera-Cabral, R. Lazkoz, R. Maartens, Phys. Rev. D 78 (2008) 023505; G. Olivares, F. Atrio-Barandela and D. Pavon, Phys. Rev. D 74 (2006) 043521; S. B. Chen, B. Wang, J. L. Jing, Phys.Rev. D 78 (2008) 123503.

[3] Sandro Micheletti, Elcio Abdalla, Bin Wang, Phys. Rev. D79 (2009) 123506; Sandro M.R. Micheletti, JCAP 1005 (2010) 009.

[4] J. Valiviita, E. Majerotto, R. Maartens, JCAP $\mathbf{0 7}$ (2008) 020, arXiv:0804.0232 J. H. He, B. Wang, E. Abdalla, Phys. Lett. B 671 (2009) 139, arXiv:0807.3471; P. Corasaniti, Phys. Rev. D 78 (2008) 083538; B. Jackson, A. Taylor, A. Berera, Phys. Rev. D79 (2009) 043526; D. Pavon, B. Wang, Gen. Relav. Grav. 41 (2009) 1; B. Wang, C.-Y. Lin, D. Pavon, E. Abdalla, Phys. Lett. B 662 (2008) 1.

[5] B. Wang, J. Zang, C.-Y. Lin, E. Abdalla and S. Micheletti, Nucl. Phys. B $\mathbf{7 7 8}$ (2007) 69; W. Zimdahl, Int. J. Mod. Phys. D 14 (2005) 2319; Z. K. Guo, N. Ohta and S. Tsujikawa, Phys. Rev. D 76 (2007) 023508; C. Feng, B. Wang, E. Abdalla, R. K. Su, Phys. Lett. B 665 (2008) 111; J. Valiviita, R. Maartens, E. Majerotto, Mon. Not. Roy. Astron. Soc. 402 (2010 2355; J. Q. Xia, Phys.Rev. D 80 (2009) 103514; M. Martinelli, L. Honorez, A. Melchiorri, O. Mena Phys. Rev. D81 (2010) 103534; L. Honorez, B. Reid, O. Mena, L. Verde, R. Jimenez, JCAP 1009 (2010) 029.

[6] J.H. He, B. Wang, P. Zhang, Phys. Rev. D 80 (2009) 063530, arXiv:0906.0677; J. H. He, B. Wang, E. Ab- dalla, Phys. Rev. D83 (2011) 063515, arXiv:1012.3904, X.D.Xu, J.H.He, B.Wang, Phys. Lett.B 701 (2011) 513, arXiv:1103.2632

[7] J.H. He, B. Wang, JCAP 06 (2008) 010, arXiv:0801.4233

[8] J. H. He, B. Wang, Y. P. Jing, JCAP 07 (2009) 030, arXiv:0902.0660

[9] G. Caldera-Cabral, R. Maartens, B. Schaefer, JCAP 0907 (2009) 027.

[10] F. Simpson, B. Jackson, J. A. Peacock, arXiv: 1004.1920; J. H. He, B. Wang, E. Abdalla, D. Pavon, JCAP 1012 (2010) 022 , arXiv: 1001.0079.

[11] O. Bertolami, F. Gil Pedro and M. Le Delliou, Phys. Lett. B 654 (2007) 165. O. Bertolami, F. Gil Pedro and M. Le Delliou, Gen. Rel. Grav. 41 (2009) 28392846, arXiv:0705.3118

[12] E. Abdalla, L.Raul W. Abramo, L. Sodre Jr., B. Wang, Phys. Lett. B673 (2009) 107; E. Abdalla, L. Abramo, J. Souza, Phys. Rev. D82 (2010) 023508, arXiv:0910.5236.

[13] P.G. Bergmann, Int. J. Theor. Phys., 1 (1968) 2536; B. N. Breizman, V. T. Gurovich, and V. P. Sokolov, Zh. Eksp. Teor. Fiz., 59 (1970) 288, Sov. Phys. JETP 32 (1971) 155; H.A.Buchdahl, Mon. Not. R. Astron. Soc. 150 (1970) 18; T.V. Ruzmaikina, and A.A. Ruzmaikin, Zh. Eksp. Teor. Fiz. 57 (1969) 680, Sov. Phys. JETP 30 (1970) 372.

[14] A. Felice, S. Tsujikawa, Living Rev. Rel. 13 (2010) 3, arXiv:1002.4928

[15] J. H. He, B. Wang, E. Abdalla, Phys. Rev. D, to appear, arXiv:1109.1730 [gr-qc].

[16] N. Dalal, K. Abazajian, E. Jenkins, A. V. Manohar, Phys. Rev. Lett 86,(2001) 1939.

[17] W. Zimdahl, D. Pavn, Gen. Rel. Grav. 35,(2003) 413.

[18] J. Wess and B. Zumino, Nucl. Phys. B70 (1974) 39-50.

[19] Sidney R. Coleman Phys. Rev. D15 (1977) 2929-2936; Curtis G. Callan, Jr., Sidney R. Coleman Phys. Rev. D16 (1977) 1762-1768.

[20] S. Coleman, F. De Luccia, Phys. Rev. D21 (1980) 33053315.

[21] A. Linde, Contemp. Concepts Phys 5 (2005) 1-362. 